\title{
Minimal fibrations of dendroidal sets
}

\author{
IEKE MOERDIJK \\ JOOST NUITEN
}

\begin{abstract}
We prove the existence of minimal models for fibrations between dendroidal sets in the model structure for $\infty$-operads, as well as in the covariant model structure for algebras and in the stable one for connective spectra. We also explain how our arguments can be used to extend the results of Cisinski (2014) and give the existence of minimal fibrations in model categories of presheaves over generalized Reedy categories of a rather common type. Besides some applications to the theory of algebras over $\infty$-operads, we also prove a gluing result for parametrized connective spectra (or $\Gamma$-spaces).
\end{abstract}

55R65, 55U35, 55P48; 18D50

\section{Introduction}

A classical fact in the homotopy theory of simplicial sets - tracing back to J C Moore's lecture notes from 1955-56 - says that any Kan fibration between simplicial sets is homotopy equivalent to a fiber bundle; see eg Barratt, Gugenheim and Moore [1], Gabriel and Zisman [13], or May [17]. This is proven by deforming a fibration onto a so-called minimal fibration, a Kan fibration whose only self-homotopy equivalences are isomorphisms. Such minimal fibrations provide very rigid models for maps between simplicial sets — in particular, they are all fiber bundles — which are especially suitable for gluing constructions.

Essentially, the same method allows one to construct minimal categorical fibrations between $\infty$-categories as well; cf Joyal [15] and Lurie [16]. In fact, these two constructions are particular cases of a general statement on the existence of minimal fibrations in certain model structures on presheaves over Reedy categories, proved by Cisinski in [7]. The case of dendroidal sets is not covered by this result however, due to the presence of nontrivial automorphisms in the base category $\Omega$.

The aim of this note is to show that the basic theory of minimal fibrations extends naturally to the setting of dendroidal sets. We say that an operadic fibration $p: Y \rightarrow X$ 
of dendroidal sets (see Cisinski and Moerdijk [8]) is minimal if all weak equivalences over $X$,

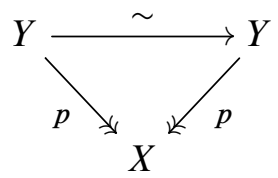

are isomorphisms. This terminology is justified by the fact that any trivial cofibration from another fibration into the fibration $p$,

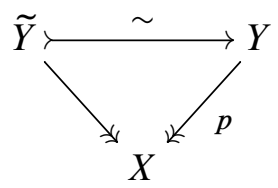

is an isomorphism. Indeed, any such trivial cofibration $i$ admits a retraction $r$ with the property that the composite $i r: Y \rightarrow Y$ is a self-weak equivalence of $Y$ over $X$, and therefore, an isomorphism.

The presence of nontrivial automorphisms in $\Omega$ makes the discussion of minimal fibrations a bit more delicate. For instance, the pullback of a minimal fibration need no longer be minimal again; see Remark 3.11 below. Our main result asserts that an operadic fibration can nonetheless be retracted onto a weakly equivalent minimal fibration, although Quillen's argument [22] showing that this retraction is a trivial fibration no longer applies in general:

Theorem 1.1 Let $p: Y \rightarrow X$ be an operadic fibration between dendroidal sets with normal domain. Then the following hold:

(a) $p$ admits a minimal fibration $M \rightarrow X$ as a fiberwise strong deformation retract.

(b) The retraction $r: Y \rightarrow M$ is a trivial fibration of dendroidal sets when the codomain $X$ is normal.

The proof of this theorem appears in Section 3 and proceeds by induction along the skeletal filtration of the domain $Y$, analogously to the classical case of simplicial sets.

One may also find minimal models for the fibrations in the covariant model structure and the stable model structure (see Bašić and Nikolaus [3]) on dendroidal sets, or any other left Bousfield localization of the operadic model structure on dendroidal sets. Indeed, any fibration $p: Y \rightarrow X$ in a left Bousfield localization of the operadic model structure is, in particular, an operadic fibration. The associated minimal operadic fibration is a retract of $p$, and therefore, a local fibration. It is minimal in the localized 
model structure since the local weak equivalences between local fibrations over $X$ coincide with the operadic weak equivalences.

The same argument shows that any left fibration $Y \rightarrow X$ of dendroidal sets admits a minimal model. Such a left fibration is not quite a fibration in a certain model category, but instead it defines a fibrant object in the covariant model structure on the over-category $\mathbf{d S e t} / X$. This model structure has been constructed in the work of Heuts [14], where it is also shown to be Quillen equivalent to the model category of algebras (in sSet) over the simplicial operad associated to $X$.

A map $f: X \rightarrow X^{\prime}$ between dendroidal sets induces a Quillen pair between the covariant model structures,

$$
f_{!}: \mathbf{d S e t} / X \rightleftarrows \mathbf{d S e t} / X^{\prime}: f^{*},
$$

which is a Quillen equivalence whenever $f$ is an operadic weak equivalence [14, Proposition 2.4]. As such, one obtains a functor

$$
\text { Alg: } \text { dSet }^{\text {op }} \rightarrow \text { ModelCat }^{\mathrm{R}}, \quad X \mapsto(\mathbf{d S e t} / X)^{\text {cov }},
$$

taking values in model categories with right Quillen functors between them. We use the theory of minimal fibrations to prove the following:

Proposition 1.2 The functor Alg preserves homotopy pullbacks. More precisely, for any diagram of dendroidal sets $X_{1} \leftarrow X_{0} \rightarrow X_{2}$ in which both arrows are cofibrations, the natural adjoint pair

colim: dSet $/ X_{1} \times_{\text {dSet } / X_{0}}^{h}$ dSet $/ X_{2} \rightleftarrows$ dSet $/ X_{1} \cup_{X_{0}} X_{2}$ :pullback

establishes a Quillen equivalence between the homotopy pullback model structure and the covariant model structure on dSet $/ X_{1} \cup_{X_{0}} X_{2}$.

Informally, this proposition asserts that algebras over a homotopy pushout of $\infty-$ operads can equivalently be described as (homotopy) matching triples of algebras over the individual pieces of the homotopy pushout. We will explain and prove Proposition 1.2 in Section 4, where we also use the theory of minimal left fibrations to give an elementary proof of a result by Heuts [14] about weak equivalences between left fibrations.

In Section 5, we briefly discuss how the arguments of the present paper yield a general existence theorem for minimal fibrations over a large class of so-called generalized Reedy categories, providing a common generalization of Cisinski's result for strict Reedy categories [7] and ours for dendroidal sets. As an application of this extended result, we have included a gluing result for parametrized connective spectra, analogous to Proposition 1.2. 
Acknowledgements We thank the referee for a careful reading and various useful comments which helped improve the exposition. Nuiten was supported by NWO.

\section{Preliminaries on dendroidal sets}

Recall that the category dSets of dendroidal sets is the category of set-valued presheaves on the category $\Omega$ of finite rooted trees; see Moerdijk and Weiss $[18 ; 19 ; 20]$. The category $\Omega$ comes equipped with two wide subcategories $\Omega^{+}$and $\Omega^{-}$whose intersection $\Omega^{+} \cap \Omega^{-}$consists of the isomorphisms in $\Omega$. The maps in $\Omega^{-}$are given by finite compositions of isomorphisms and degeneracy maps, ie maps $\sigma_{v}: T \rightarrow T \backslash v$ obtained by picking a vertex $v$ of $T$ with a single input, removing that vertex and identifying the incoming and outgoing edges. Similarly, the maps in $\Omega^{+}$are given by finite compositions of isomorphisms and the following two kinds of face maps:

(1) for every inner edge $e$ of a tree $T$, ie an edge connecting two vertices, an inner face map $\partial_{e}: T / e \rightarrow T$ obtained by contracting $e$, and

(2) for every external vertex $v$ of a tree $T$, ie a vertex with an adjacent edge $e$ such that all other adjacent edges are outer edges, an outer face map $\partial_{v}: T / v \rightarrow T$ obtained by removing $v$, as well as all edges attached to it except $e$. Note that the edge $e$ is the unique inner edge attached to $v$, except when the tree $T$ is an $n$-corolla $C_{n}$. In the latter case, there are $n+1$ outer face maps, one associated to each edge of $C_{n}$.

For any finite rooted tree $T$, the degree of $T$ is given by the number of vertices of $T$. It is immediate that noninvertible arrows in $\Omega^{+}$(resp. $\Omega^{-}$) raise (resp. lower) the degree. Furthermore, every map in $\Omega$ factors essentially uniquely as a map in $\Omega^{-}$, followed by a map in $\Omega^{+}$. Altogether, this gives the category $\Omega$ the structure of a (generalized) Reedy category; see Berger and Moerdijk [5].

Lemma 2.1 Any map in $\Omega^{-}$is a split epimorphism, and two maps $\sigma, \tau: T \rightarrow S$ in $\Omega^{-}$are the same if they have the same set of sections.

Proof Observe that a degeneracy $\sigma_{v}: T \rightarrow T \backslash v$ admits precisely two sections, obtained by choosing an edge above or below the vertex $v$ and considering the face map induced by contracting this edge. This immediately implies that all maps in $\Omega^{-}$ are split epimorphisms.

A map of trees is completely determined by its effect on the set of edges, so the sections of a map $\sigma: T \rightarrow S$ in $\Omega^{-}$form a subset of the set of sections of the induced surjection 
$\sigma_{*}: \operatorname{Edge}(T) \rightarrow \operatorname{Edge}(S)$. On the other hand, any section $i$ of $\sigma_{*}$ is induced by the iterated face map $\delta: S \rightarrow T$ that contracts all edges of $T$ which are not contained in the image of $i$. This face map is a section since $\sigma \delta$ induces the identity map on colors. It follows that sections of a map $\sigma$ in $\Omega^{-}$correspond bijectively to sections of the associated surjection between sets of edges. Since surjections of sets are uniquely determined by their sets of sections, we conclude that the maps in $\Omega^{-}$are uniquely determined by their sections.

Recall that a pushout square is called absolute if every functor (or equivalently, the Yoneda embedding) sends it to a pushout square. The following lemma is taken from [18]. For completeness we give a compact replacement of the proof given in [loc. cit.].

Lemma 2.2 Any pair of maps $\sigma: S \rightarrow S^{\prime}, \tau: S \rightarrow T$ in $\Omega^{-}$fits into an absolute pushout square

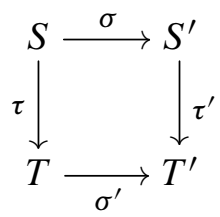

in which $\sigma^{\prime}$ and $\tau^{\prime}$ are in $\Omega^{-}$as well.

Proof Since absolute pushout squares can be pasted, it suffices to check this when $\sigma=\sigma_{v}: S \rightarrow S \backslash v$ and $\tau=\sigma_{w}: S \rightarrow S \backslash w$ are degeneracy maps. In this case, one can easily check that the required pushout square (1) can be produced by taking $T^{\prime}=S \backslash\{v, w\}$ and $\sigma^{\prime}$ (resp. $\tau^{\prime}$ ) the degeneracy removing the vertex $v$ (resp. $w$ ) and identifying the ingoing and outgoing edge. In the case where $v=w$, the maps $\sigma^{\prime}$ and $\tau^{\prime}$ are simply the identity maps.

To see that the resulting pushout square is an absolute pushout square, it suffices to find sections $\alpha$ of $\sigma$ and $\alpha^{\prime}$ of $\sigma^{\prime}$ which are compatible in the sense that $\tau \alpha=\alpha^{\prime} \tau^{\prime}$; see eg van den Berg and Moerdijk [4]. When the vertices $v$ and $w$ are the same, one can just pick any section of $\sigma=\sigma_{v}$ and take the identity section of $\sigma^{\prime}=$ id. If $v$ is different from $w$ and $v$ is not connected to $w$ by a single edge, one can take both $\alpha$ and $\alpha^{\prime}$ to be the face map contracting the edge below the vertex $v$ (seen as a vertex in $S$ and in $S \backslash w$, respectively).

We are left with the case that the vertices $v$ and $w$ are connected by a single edge. If $v$ is the vertex directly above $w$, compatible sections are provided by letting $\alpha$ and $\alpha^{\prime}$ be the face maps contracting the edge above $v$ (again seen as a vertex in $S$ and in $S \backslash w$, respectively). If $v$ is the vertex directly under $w$, one can take $\alpha$ and $\alpha^{\prime}$ to be the face maps contracting the edge below $v$. 
We identify elements of a dendroidal set $X$ with maps $x: \Omega[T] \rightarrow X$, where $\Omega[T]$ is the presheaf represented by the tree $T$. An element $x: \Omega[T] \rightarrow X$ is called degenerate if it factors as $\Omega[T] \rightarrow \Omega[S] \rightarrow X$, where $T \rightarrow S$ is a noninvertible map in $\Omega^{-}$. It follows easily from Lemma 2.2 that any element of a dendroidal set decomposes essentially uniquely as a map $\Omega[T] \rightarrow \Omega[S]$ in $\Omega^{-}$, followed by a nondegenerate element $\Omega[S] \rightarrow X$; see eg [5, Proposition 6.7].

For every tree $T$, there is an action of the automorphism group $\operatorname{Aut}(T)$ on the set of nondegenerate elements $\Omega[T] \rightarrow X$. If $x: \Omega[T] \rightarrow X$ is an element of $X$, define its automorphism group $\operatorname{Aut}(x) \subseteq \operatorname{Aut}(T)$ to be the isotropy group of the element $x$ under this action. A map of dendroidal sets $f: X \rightarrow Y$ induces a (necessarily injective) map $\operatorname{Aut}(x) \rightarrow \operatorname{Aut}(f x)$.

A monomorphism $i: A \rightarrow B$ between dendroidal sets is called normal if a nondegenerate element of $B$ has a trivial automorphism group whenever it does not factor through $i$. In other words, $\operatorname{Aut}(T)$ acts freely on the set of nondegenerate elements in $B(T) \backslash A(T)$. A dendroidal set $X$ is called normal if the map $\varnothing \rightarrow X$ is a normal monomorphism. If $B$ is a normal dendroidal set, any monomorphism $A \rightarrow B$ is normal.

Remark 2.3 In fact, for a normal monomorphism $i: A \rightarrow B$, the group $\operatorname{Aut}(T)$ acts freely on the set of all elements $\Omega[T] \rightarrow B$ that do not factor through $i$, by Cisinski and Moerdijk [8, Proposition 1.5]. This implies that any map with a normal codomain also has a normal domain.

\subsection{Skeletal filtration}

Let $t_{n}: \Omega_{\leq n} \rightarrow \Omega$ be the inclusion of the full subcategory of $\Omega$ on the objects of degree $\leq n$. The $n$-skeleton of a dendroidal set $X$ is given by $X^{(n)}:=t_{n} ! t_{n}^{*} X$. The skeleta of $X$ fit into a natural skeletal filtration

$$
\varnothing=X^{(-1)} \rightarrow X^{(0)} \rightarrow X^{(1)} \rightarrow \cdots \rightarrow X .
$$

Because every element of a dendroidal set $X$ factors in an essentially unique way as a map in $\Omega^{-}$, followed by a nondegenerate element, the maps in the skeletal filtration (2) are all monomorphisms, and the colimit of this sequence of inclusions is the original dendroidal set $X$. Indeed, $X^{(n)}$ is the subobject of $X$ consisting of those elements $\Omega[T] \rightarrow X$ that factor through some tree $S$ of degree $\leq n$. For example, the boundary $\partial \Omega[T]$ of a representable presheaf is defined as the $(n-1)$-skeleton $\Omega[T]^{(n-1)}$, where $n$ is the degree of the tree $T$. Explicitly, $\partial \Omega[T](S)$ is the set of maps $S \rightarrow T$ in $\Omega$ that factor through a face map (up to isomorphism).

When $x: \Omega[T] \rightarrow X$ is an element of $X$, define the boundary $\partial x$ of $x$ to be the restriction of $x$ to $\partial \Omega[T]$. The following is a straightforward variation of [7, Lemma 2.6]: 
Lemma 2.4 Let $x, y: \Omega[T] \rightarrow X$ be two degenerate elements of a normal dendroidal set $X$. If the boundaries of $x$ and $y$ agree, then $x$ and $y$ are the same.

Proof Write $x=\sigma^{*} \bar{x}$ and $y=\tau^{*} \bar{y}$ where $\bar{x}: \Omega[S] \rightarrow X$ and $\bar{y}: \Omega\left[S^{\prime}\right] \rightarrow X$ are nondegenerate and $\sigma: T \rightarrow S$ and $\tau: T \rightarrow S^{\prime}$ are noninvertible maps in $\Omega^{-}$. Let $\alpha$ be any section of $\sigma$ and let $\beta$ be any section of $\tau$. Since the boundaries of $x$ and $y$ agree, we have that

$$
\bar{x}=\alpha^{*} x=\alpha^{*} y=(\tau \alpha)^{*} \bar{y},
$$

and similarly, $\bar{y}=(\sigma \beta)^{*} \bar{x}$. If the composite map $\tau \alpha$ in $\Omega$ could be factored as a noninvertible map in $\Omega^{-}$, followed by a map in $\Omega^{+}$, then $\bar{x}$ would be a degenerate element. In other words, the map $\tau \alpha: S \rightarrow T \rightarrow S^{\prime}$ is contained in $\Omega^{+}$, and in particular, the degree of $S$ is less than or equal to the degree of $S^{\prime}$. Applying the same argument to the composite $\sigma \beta$ shows that the degrees of $S$ and $S^{\prime}$ agree, which in turn implies that $\tau \alpha$ and $\sigma \beta$ are isomorphisms. Furthermore, we have that

$$
(\tau \alpha)^{*}(\sigma \beta)^{*} \bar{x}=(\tau \alpha)^{*} \bar{y}=\bar{x} .
$$

Since $\bar{x}$ is a nondegenerate element of a normal dendroidal set, it has no nontrivial automorphisms. From this, we conclude that

( $\star$ for any choice of sections $\alpha \in \Gamma(\sigma)$ and $\beta \in \Gamma(\tau)$, the map $\sigma \beta$ is inverse to $\tau \alpha$. We claim that $\sigma=\sigma \beta \tau$, in which case, we conclude that

$$
y=\tau^{*} \bar{y}=\tau^{*}(\sigma \beta)^{*} \bar{x}=\sigma^{*} \bar{x}=x .
$$

To see that $\sigma=\sigma \beta \tau$, it suffices to check that both maps in $\Omega^{-}$have the same set of sections, by Lemma 2.1. Our conclusion ( $\star$ ) shows that $\alpha$ is a section of $\sigma \beta \tau$ as soon as $\alpha$ is a section of $\sigma$. For the converse, suppose that $\alpha$ is a section of $\sigma \beta \tau$. Since $\sigma \beta$ is an isomorphism, we have that $\tau \alpha$ is an inverse to $\sigma \beta$ and consequently $\alpha \sigma \beta$ is a section of $\tau$.

But now observe that $(\star)$ implies that the isomorphism $\sigma \beta$ is actually independent of the chosen section $\beta \in \Gamma(\tau)$. This means that $\sigma \beta=\sigma(\alpha \sigma \beta)$, which in turn implies that $\alpha$ is a section of $\sigma$.

When $X$ is a normal dendroidal set, the skeletal filtration (2) can be obtained by attaching cells [5]. More precisely, each inclusion $X^{(n-1)} \rightarrow X^{(n)}$ fits into a pushout square:

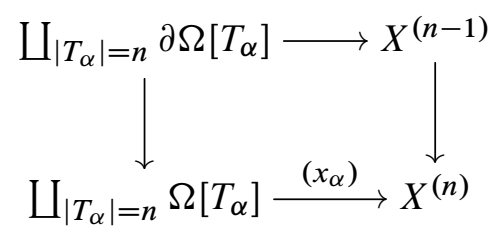


We will call the resulting elements $x_{\alpha}: \Omega\left[T_{\alpha}\right] \rightarrow X$ generating nondegenerate elements. For every nondegenerate element $x$ of $X$ there is a unique generating nondegenerate element $x_{\alpha}$, together with a unique automorphism $\phi$ of $T_{\alpha}$, such that $x=\phi^{*} x_{\alpha}$.

\subsection{Tensor product}

The category $\Omega$ can be seen as a full subcategory of the category Oper of operads in sets [19]. The inclusion of $\Omega$ in Oper induces a functor $N:$ Oper $\rightarrow \mathbf{d S e t}$ called the dendroidal nerve, with left adjoint $\tau$. The dendroidal nerve extends the usual simplicial nerve of a category, in the sense that it fits into a commuting diagram:

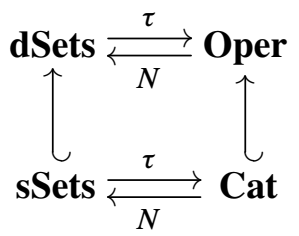

Recall that the category Oper of operads in sets carries a monoidal structure given by the Boardman-Vogt tensor product $\otimes_{B V}$. This induces a tensor product $\otimes$ on the category of dendroidal sets by defining

$$
X \otimes Y=N\left(\tau X \otimes_{B V} \tau Y\right)
$$

for representable $X$ and $Y$, and by requiring that $\otimes$ preserves colimits in each of its variables. We will mainly need the following pushout-product property of the tensor product (which is not part of a monoidal structure): for a normal monomorphism $A \rightarrow B$ of dendroidal sets and a monomorphism of simplicial sets $K \rightarrow L$, the induced map of dendroidal sets,

$$
L \otimes A \amalg_{K \otimes A} K \otimes B \longrightarrow L \otimes B,
$$

is again a normal monomorphism [8, Proposition 1.9]; see also [10].

\subsection{Model structures}

The category of dendroidal sets admits a left proper model structure (called the operadic model structure) which is Quillen equivalent to the model structure on simplicial operads; see Cisinski and Moerdijk [8; 9]. The cofibrations of this model structure are given by the normal monomorphism and the fibrant objects are given by the $\infty$-operads, which are defined by extension properties with respect to certain dendroidal inner horn inclusions. More details on horn inclusions in the setting of dendroidal sets will be given when we need them in Section 4. 
We will call the fibrations in this model structure operadic fibrations. To get some feeling for their behavior, denote by $J$ the dendroidal nerve of the groupoid $\{0 \simeq 1\}$ with objects 0 and 1 , together with a unique isomorphism between them. The operadic fibrations then have the right lifting property with respect to the pushout-product map

$$
J \otimes A \cup\{i\} \otimes A\{i\} \otimes B \longrightarrow J \otimes B,
$$

where $i=0,1$ and $A \rightarrow B$ is a normal monomorphism. Stated differently, the above map is a trivial cofibration for all normal monomorphisms $A \rightarrow B$. Conversely, by [8], a map between $\infty$-operads is an operadic fibration if and only if it has the right lifting property against all maps (5) induced by normal monomorphisms $A \rightarrow B$, as well as the right lifting property with respect to the inner horn inclusions.

There are two other model structures on the category of dendroidal sets, obtained from the operadic model structure by left Bousfield localization. The covariant model structure, constructed by Heuts [14], has fibrant objects given by those $\infty$-operads that also have the extension property against certain left horn inclusions, and is Quillen equivalent to the model category of $E_{\infty}$-algebras (in spaces). The stable model structure of Bašić and Nikolaus [3] is a further Bousfield localization of the covariant model structure, whose fibrant objects have the extension property with respect to all horn inclusions. This model category is equivalent to the model category of grouplike $E_{\infty}$-spaces, or equivalently, connective spectra.

Replacing the terminal dendroidal set by an arbitrary dendroidal set $X$, one can also obtain the covariant model structure on $\mathbf{d S e t} / X$ as a Bousfield localization of the operadic model structure. This model structure has fibrant objects given by the left fibrations $Y \rightarrow X$, which are characterized by the right lifting property against the left horn inclusions. It is equivalent to the model category of algebras (in spaces) over the simplicial operad corresponding to $X$ under the aforementioned Quillen equivalence between dendroidal sets (operadically) and simplicial operads.

\subsection{Cylinders and homotopies}

Taking the tensor product of $X$ with the functors $\{0,1\} \rightarrow J \rightarrow *$ provides a factorization of the fold map

$$
X \amalg X \simeq\{0,1\} \otimes X \longrightarrow J \otimes X \stackrel{\sigma}{\rightarrow} X .
$$

When $X$ is a normal dendroidal set, the first map is a cofibration and the second map is an operadic weak equivalence, by the pushout-product properties (4) and (5). The functor $J \otimes(-)$ therefore provides a cylinder construction for normal dendroidal sets; furthermore, this construction preserves colimits. 
The cylinder $J \otimes(-)$ induces the usual variants of the notion of homotopy. For example, let $p: Y \rightarrow X$ be a map and let $y_{0}, y_{1}: \Omega[T] \rightarrow Y$ be two elements of $Y$. Then a fiberwise homotopy between $y_{0}$ and $y_{1}$, relative to the boundary $\partial \Omega[T]$, is given by a map $H$ which fits into a commuting diagram

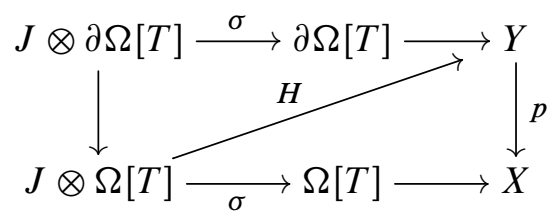

whose restriction to $\{i\} \otimes \Omega[T]$ agrees with the map $y_{i}$ (for $i=0,1$ ).

Lemma 2.5 Let $p: Y \rightarrow X$ be an operadic fibration. Then fiberwise homotopy relative to the boundary provides an equivalence relation on the set of elements $Y(T)$. More generally, for any monomorphism $A \rightarrow B$ between normal dendroidal sets, the notion of fiberwise homotopy relative to $A$ provides an equivalence relation on $\operatorname{Hom}(B, Y)$.

Proof This is a standard argument using the homotopy extension and lifting property. For later reference (cf the proof of Proposition 3.3), we prove transitivity in a slightly more general setting. Let $x, y, z: B \rightarrow Y$ be maps and suppose that there are fiberwise homotopies $g: x \simeq y$ and $h: y \simeq z$, where $h$ is a fiberwise homotopy rel $A$. Then $x \simeq z$ via a homotopy that agrees with $g$ when restricted to $A$. Indeed, consider the following diagram:

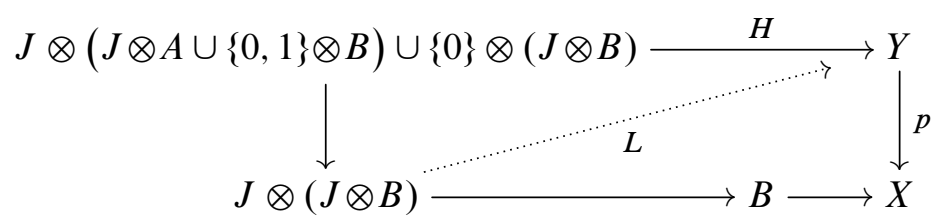

In this diagram, the map $H$ is given heuristically by $H(s, t, a)=g(t, a)$ on $J \otimes(J \otimes A)$, while it is given on $J \otimes(\{0,1\} \otimes B) \cup\{0\} \otimes(J \otimes B)$ by

$$
H(s, 0, b)=x(b), \quad H(s, 1, b)=h(s, b), \quad H(0, t, b)=g(t, b) .
$$

The left vertical map is of the form (5) for the normal monomorphism

$$
J \otimes A \amalg_{\{0,1\} \otimes A}\{0,1\} \otimes B \longrightarrow J \otimes B,
$$

which is itself of the form (4). It follows that the left vertical map is a trivial cofibration, so there is a lift $L$ as indicated since $p$ was an operadic fibration. The restriction of $L$ to $\{1\} \otimes(J \otimes B)$ provides a fiberwise homotopy between $x$ and $z$, which agrees with $g$ when restricted to $A$. 


\section{Existence of minimal fibrations}

This section contains the proof of Theorem 1.1, which asserts that any fibration of dendroidal sets $Y \rightarrow X$ admits a minimal fibration as a deformation retract, at least when $Y$ is normal. The idea of the proof is to construct a deformation retract of the fibration $Y \rightarrow X$ which is skeletal (see Definition 3.1) by induction over the skeletal filtration of $Y$. We then show that any such skeletal fibration is a minimal fibration.

\subsection{Skeletal fibrations}

The following definition is an immediate analogue of the notion of "skeletality" appearing in the classical literature on simplicial sets (where it is usually called minimality, anticipating Corollary 3.7):

Definition 3.1 Let $p: Y \rightarrow X$ be an operadic fibration of dendroidal sets. We will say that $p$ is a skeletal fibration if for any two elements $y_{0}, y_{1}: \Omega[T] \rightarrow Y$ which are fiberwise homotopic relative to their boundary, there is an automorphism $\phi \in \operatorname{Aut}(T)$ such that $y_{0}=\phi^{*} y_{1}$.

There is a second natural extension of the notion of "skeletality" to dendroidal sets, where one requires two homotopic elements to be equal. This condition is too restrictive for our purposes. Indeed, the following example demonstrates that there are dendroidal sets that cannot have a deformation retract satisfying this stricter condition of skeletality:

Example 3.2 Let $C_{2}$ be the 2-corolla, and let $\eta$ be the tree with a single edge and no vertices. Their associated dendroidal sets are $\Omega\left[C_{2}\right]$ and $\Delta[0]:=\Omega[\eta]$. The $2-$ corolla $C_{2}$ has a single nontrivial automorphism $\tau$ of order 2 , and its boundary $\partial \Omega\left[C_{2}\right]$ is the disjoint union of three edges. Define $J \otimes_{\tau} \Omega\left[C_{2}\right]$ to be the pushout:

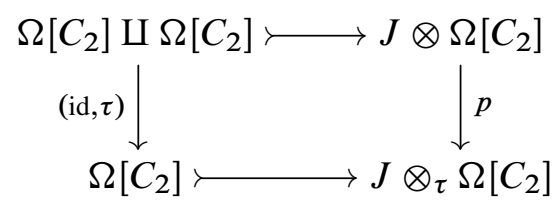

The bottom map defines an element of $J \otimes_{\tau} \Omega\left[C_{2}\right]$ which is $J$-homotopic to its conjugate by $\tau$. On the other hand, $J \otimes_{\tau} \Omega\left[C_{2}\right]$ is normal since the top map in this pushout diagram is a normal monomorphism and $\Omega\left[C_{2}\right]$ is normal. Next, consider the pushout:

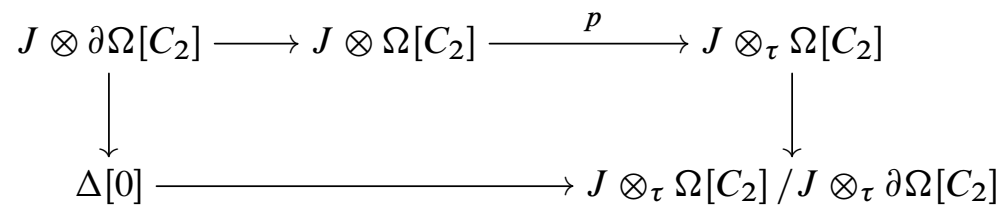


Note that both $J \otimes \partial \Omega\left[C_{2}\right]$ and $\Delta[0]$ have no elements indexed by nonlinear trees. Since pushouts of dendroidal sets are computed objectwise, this implies that the pushout $J \otimes_{\tau} \Omega\left[C_{2}\right] / J \otimes_{\tau} \partial \Omega\left[C_{2}\right]$ is again a normal dendroidal set. Finally, let

$$
J \otimes_{\tau} \Omega\left[C_{2}\right] / J \otimes_{\tau} \partial \Omega\left[C_{2}\right] \stackrel{\sim}{\longrightarrow} X
$$

be a fibrant-cofibrant replacement of this dendroidal set. The composite

$$
x: \Omega\left[C_{2}\right] \longrightarrow J \otimes_{\tau} \Omega\left[C_{2}\right] \longrightarrow J \otimes_{\tau} \Omega\left[C_{2}\right] / J \otimes_{\tau} \partial \Omega\left[C_{2}\right] \longrightarrow X
$$

defines an element $x$ of $X$ with the property that $x$ is homotopic (relative to the boundary) to $\tau^{*} x$, while $x$ differs from $\tau^{*} x$ since $X$ was assumed normal. This property is shared by the image of $x$ under a retraction $r: X \rightarrow M$. We conclude that any retraction of $X$ admits two distinct (but conjugate) 2-corollas which are homotopic relative to their boundary.

Proposition 3.3 Let $p: Y \rightarrow X$ be a fibration of dendroidal sets with normal domain. Then $p$ has a skeletal fibration $q: M \rightarrow X$ as a fiberwise strong deformation retract (with respect to the functorial cylinder $J$ ).

Proof We construct the inclusion $i: M \subseteq Y$, the retraction $r: Y \rightarrow M$ and the strong deformation retraction $H: J \otimes Y \rightarrow Y$ all at the same time, by induction along the skeleta of $Y$. Suppose that we have formed

$$
M^{(n)} \underset{r^{(n)}}{\stackrel{i^{(n)}}{\rightleftarrows}} Y^{(n)} \quad \text { and } \quad J \otimes Y^{(n)} \stackrel{H^{(n)}}{\longrightarrow} Y
$$

where $H_{0}^{(n)}$ is the inclusion of $Y^{(n)}$ into $Y$, and $H_{1}^{(n)}$ is the composite $i^{(n)} \circ r^{(n)}$. All maps are maps over the base $X$, where $M^{(n)}$ is considered as the domain of the map $p i^{(n)}: M^{(n)} \rightarrow Y \rightarrow X$.

We start by producing $M^{(n+1)}$ and the inclusion $i^{(n+1)}: M^{(n+1)} \rightarrow Y^{(n+1)}$. Recall that $Y^{(n+1)}$ is obtained from $Y^{(n)}$ by attaching a set of generating nondegenerate elements (together with their conjugates under the $\operatorname{Aut}(T)$-action). For each $T \in \Omega$ of degree $n+1$, the set of generating nondegenerate $y: \Omega[T] \rightarrow Y$ decomposes as the disjoint union of the following three subsets of $Y(T)$ :

(A) those $y$ whose boundary takes values in $M^{(n)}$ and which are not fiberwise homotopic (relative to the boundary) to a degenerate element in $M^{(n)}$,

(B) those $y$ which are fiberwise homotopic (relative to the boundary) to a degenerate element of $M^{(n)} \subseteq Y$, and

(C) those $y$ whose boundary $\partial y: \partial \Omega[T] \rightarrow Y$ takes no values in the subobject $M^{(n)}$. 
The elements in $C$ cannot be attached to $M^{(n)}$ and the elements in $B$ should not be attached to $M^{(n)}$ in the construction of $M^{(n+1)}$, since they would give rise to homotopic elements which are not related by the action of $\operatorname{Aut}(T)$.

We say that two elements $y_{0}$ and $y_{1}$ of the remaining set $A$ are equivalent if $y_{0}$ is fiberwise homotopic (relative the boundary) to $\phi^{*} y_{1}$ for some $\phi \in \operatorname{Aut}(T)$. This defines an equivalence relation by Lemma 2.5. Let $A_{M}$ be a set of representatives for each of the equivalence classes and let $A_{Y}$ be its complement in $A$.

We now construct $M^{(n+1)}$ and $i^{(n+1)}: M^{(n+1)} \rightarrow Y^{(n+1)}$ by attaching one copy of $\Omega[T]$ to $M^{(n)}$ for every equivalence class of elements in the set $A$ and mapping it to its representing element in $A_{M} \subseteq Y(T)$. This defines $M^{(n+1)}$ together with an inclusion into $Y^{(n+1)}$. This inclusion fits into the following diagram, in which the two solid squares are pushouts:

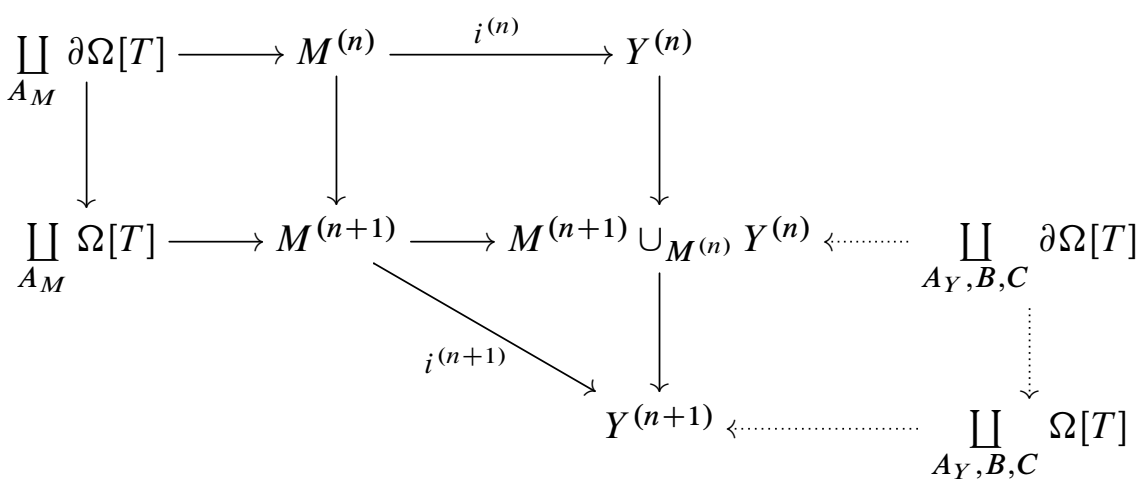

The resulting map $M^{(n+1)} \cup_{M^{(n)}} Y^{(n)} \rightarrow Y^{(n+1)}$ is obtained by attaching those cells of $Y^{(n+1)}$ that we have not yet attached in our construction of $M^{(n+1)}$. This is pictured by the dotted pushout diagram in (6), where the attaching maps indexed by the sets $A_{Y}$ and $B$ take values in $M^{(n)}$ and the ones indexed by $C$ take values in $Y^{(n)}$.

Having constructed the inclusion $M^{(n+1)} \rightarrow Y^{(n+1)}$, our next task is to extend the deformation retraction $H^{(n)}$. The constant homotopy on $M^{(n+1)}$ and the homotopy $H^{(n)}$ on $Y^{(n)}$ (relative to $M^{(n)}$ ) together define a homotopy

$$
J \otimes\left(M^{(n+1)} \cup_{M^{(n)}} Y^{(n)}\right) \longrightarrow Y .
$$

We extend this homotopy along each of the cell attachments that constitute the dotted pushout diagram in (6):

Case $\boldsymbol{A}_{\boldsymbol{Y}}$ We attach a generating nondegenerate element $y: \Omega[T] \rightarrow Y$ which is fiberwise homotopic (relative to the boundary) to the conjugate of an element in $A_{M}$. Since we constructed $M^{(n+1)}$ by attaching the elements in $A_{M}$ (and their conjugates), 
it follows that $y$ is homotopic to an element in $M^{(n+1)}$. The extension of $H^{(n)}$ to the element $y$ is given by a choice of such a fiberwise homotopy (relative boundary).

Case $\boldsymbol{B}$ We attach a generating nondegenerate element $y: \Omega[T] \rightarrow Y$ which is fiberwise homotopic (relative boundary) to a degenerate element that is contained in $M^{(n)}$ and, therefore, in $M^{(n+1)}$. The extension of $H^{(n)}$ to the element $y$ is then given by a choice of such fiberwise homotopy (relative boundary).

Case $C$ We attach a generating nondegenerate element $y: \Omega[T] \rightarrow Y$ whose boundary is not contained in $M^{(n)}$. We can use the restriction of $H^{(n)}$ to the boundary of $y$, together with the element $y$ itself, to form the following commuting diagram:

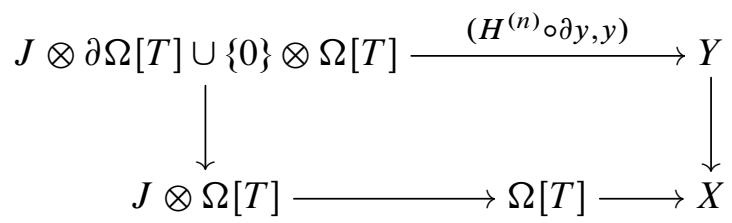

Since $Y \rightarrow X$ is a fibration, there is a lift $h: J \otimes \Omega[T] \rightarrow Y$ which provides a fiberwise homotopy between $y$ and an element $z: \Omega[T] \rightarrow Y$ whose boundary is the evaluation of $H^{(n)} \circ \partial y$ at 1 , which takes values in $M^{(n)}$. By construction, this fiberwise homotopy $h$ extends the deformation retraction $H^{(n)}$ applied to the boundary of $y$.

When $z$ is an element of $M^{(n+1)}$ we use the homotopy $h$ to extend $H^{(n)}$ to the element $y$. When $z$ is not contained in $M^{(n+1)}$, we have already constructed a homotopy $k$ (relative to the boundary) between $z$ and an element $m$ in $M^{(n+1)}$ in the previous two steps. We can compose the two homotopies $h$ and $k$ (as in the proof of Lemma 2.5) to produce a homotopy between $y$ and $m$ which agrees with $H^{(n)}$ on the boundary. Use this homotopy to extend $H^{(n)}$ over the element $y$.

In this way we produce a fiberwise strong deformation retraction

$$
H^{(n+1)}: J \otimes Y^{(n+1)} \longrightarrow Y
$$

extending $H^{(n)}$. By construction, the restriction of $H^{(n+1)}$ to $\{1\} \otimes Y^{(n+1)}$ factors as $i^{(n+1)} \circ r^{(n+1)}$ for some retraction $r^{(n+1)}: Y^{(n+1)} \rightarrow M^{(n+1)}$. Proceeding by induction on the skeleton, we thus obtain a fiberwise strong deformation retract of $Y$ onto some subobject $M$.

It remains to check that the resulting map $q=p i: M \rightarrow X$ is a skeletal fibration. Since $M$ is a fiberwise retract of $Y$, it follows that the map $q: M \rightarrow X$ is a fibration. To see that it is skeletal, let $x, y: \Omega[T] \rightarrow M$ be two elements of $M$ that are fiberwise homotopic relative to their boundary. If both maps are degenerate, then they must 
be the same since their boundaries are the same; see Lemma 2.4. We may therefore assume that $x$ is nondegenerate.

Applying the inclusion $i$, we see that $x$ and $y$ determine homotopic elements (relative boundary) in $Y$, whose boundary lies in the $n$-skeleton $M^{(n)}$. By the construction of $M^{(n+1)}$, it follows that $y$ is nondegenerate as well: indeed, we removed all nondegenerate elements from $Y$ that were fiberwise homotopic (relative boundary) to degenerate elements in $M$. But then the construction of $M$ implies that $x=\phi^{*} y$ for some $\phi \in \operatorname{Aut}(T)$, since we attached only one generating nondegenerate element to $M^{(n)}$ for each equivalence class of nondegenerate elements in $Y$.

\subsection{Minimal fibrations}

Let $p: Y \rightarrow X$ be a skeletal fibration with normal domain. To check that $p$ is a minimal fibration, it suffices to check that any fiberwise self-homotopy equivalence of $p$ is an isomorphism. In turn, this is guaranteed by the following:

Proposition 3.4 Suppose that $p: Y \rightarrow X$ is a skeletal fibration with normal domain. If $f: Y \rightarrow Y$ is an endomorphism of $p$ which is fiberwise homotopic to the identity map on $Y$, then $f$ is an isomorphism.

We prove this by induction on the skeleton of $Y$, the case $Y^{(-1)}$ being trivial. The inductive step follows from the following two lemmas:

Lemma 3.5 Let $p: Y \rightarrow X$ be a skeletal fibration with normal domain $Y$ and let $h: J \otimes Y \rightarrow Y$ be a fiberwise homotopy from an endomorphism $h_{0}$ of $p$ to the identity map. If $h_{0}$ induces an isomorphism on the $n$-skeleton $Y^{(n)}$, then $h_{0}$ is injective on the $(n+1)-$ skeleton of $Y$.

Proof Let $T \in \Omega$ be of degree $n+1$ and let $x, y: \Omega[T] \rightarrow Y$ be two elements such that $h_{0} x=h_{0} y$. We have that $p x=p y$ and, by inductive hypothesis, $\partial x=\partial y$. We may clearly assume that one of the two, say $x$, is a nondegenerate element.

Take the (fiberwise) homotopies $h(x)$ and $h(y)$ from $h_{0} x$ to $x$ and from $h_{0} y$ to $y$, together with the constant homotopies on $\partial x=\partial y$ and $h_{0}(x)=h_{0}(y)$. Together these give a map $K$ which fits into a commuting square:

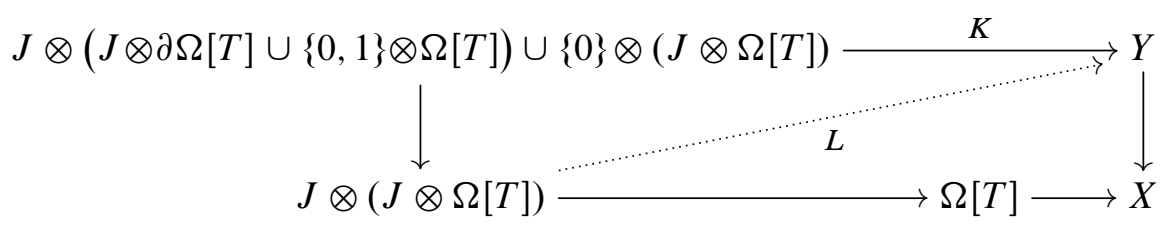


This square allows for a diagonal map $L$ because $Y \rightarrow X$ is a fibration. The composite

$$
\{1\} \otimes(J \otimes \Omega[T]) \longrightarrow J \otimes(J \otimes \Omega[T]) \stackrel{L}{\longrightarrow} Y
$$

determines a fiberwise homotopy between $x$ and $y$, relative to $\partial \Omega[T]$. The fibration $p$ is skeletal, so $x=\sigma^{*} y$ for some $\sigma \in \operatorname{Aut}(T)$. But then we also have that $h_{0}(x)=$ $\sigma^{*} h_{0}(y)=\sigma^{*} h_{0}(x)$. Since $Y$ is normal, this either means that $\sigma=1$ (if $h_{0}(x)$ is nondegenerate) or $h_{0}(x)$ is degenerate.

In the latter case, there is a degenerate $z$ such that $h_{0}(x)=h_{0}(z)$, since $h_{0}$ was assumed to be an isomorphism on $Y^{(n)}$. Repeating the previous argument shows that $x=\tau^{*} z$ for some $\tau \in \operatorname{Aut}(T)$. Since $x$ was assumed nondegenerate, this cannot happen and we conclude again that $\sigma=1$. This shows that $x=y$.

Lemma 3.6 Let $f: Y \rightarrow Y$ be a fiberwise homotopy equivalence from a skeletal fibration $p: Y \rightarrow X$ with normal domain to itself. If $f$ induces an isomorphism on $Y^{(n)}$, then $f$ induces a surjective map on elements of degree $n+1$.

Proof Let $f: Y \rightarrow Y$ be a fiberwise homotopy equivalence from $p$ to itself. Factor $f=q i$ where $i: Y \rightarrow Z$ is a cofibration and $q: Z \rightarrow Y$ is a trivial fibration. Since $Y$ is normal, so is $Z$, and $i$ is the inclusion of a fiberwise strong deformation retract over $X$, with retraction $r: Z \rightarrow Y$ over $X$.

Let $T \in \Omega$ be of degree $n+1$ and take $x: \Omega[T] \rightarrow Y$. Because $f$ induces an isomorphism on the $n$-skeleton of $Y$, there is a map $y: \partial \Omega[T] \rightarrow Y$ such that $f y=\partial x$. Since $q$ is a trivial fibration, there is a map $z: \Omega[T] \rightarrow Z$ such that $q z=x$ and $\partial z=i y$ :

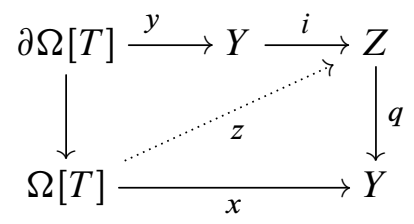

Let $w=r(z)$. Then $\partial f(w)=f r(\partial z)=f r i(y)=\partial x$, and $f(w)$ is fiberwise homotopic to $x($ rel $\partial \Omega[T])$ :

$$
f(w)=\operatorname{fr}(z)=\operatorname{qir}(z) \simeq \operatorname{rel} \partial \Omega[T] q(z)=x .
$$

Since $p$ was skeletal, it follows that $f(w)=\sigma^{*} x$ for some $\sigma \in \operatorname{Aut}(T)$, and so $x=f\left(\sigma^{-1 *} w\right)$.

Proof of Theorem 1.1 Let $p: Y \rightarrow X$ be an operadic fibration with normal domain. By Proposition 3.3, $p$ admits a skeletal fibration $q: M \rightarrow X$ as a fiberwise strong 
deformation retract, with inclusion $i: M \rightarrow Y$ and retraction $r: Y \rightarrow M$. The object $M$ is normal, being the retract of a normal object. It then follows from Proposition 3.4 that $q$ is a minimal fibration.

It remains to check that the retraction $r: Y \rightarrow M$ is a trivial fibration when the base $X$ is a normal dendroidal set. This is proven exactly as in Quillen's paper [22], which treats the analogous result for simplicial sets. Consider a diagram of the form:

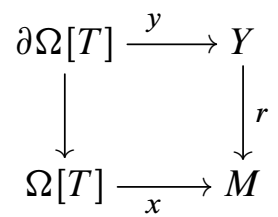

Then $i x$ provides a lift making the bottom triangle commute, but the boundary of $i x$ agrees with iry: $\partial \Omega[T] \rightarrow Y$, which is only fiberwise homotopic to $y$ using the deformation retraction $H$ between $i r$ and the identity on $Y$.

We therefore replace $i x$ by a homotopic element of $Y$ whose boundary agrees with $y$. Since $p: Y \rightarrow X$ is a fibration, there is a lift in the following diagram:

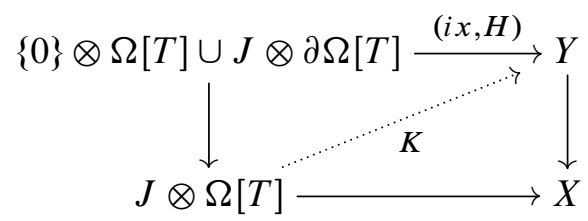

Let $z: \Omega[T] \rightarrow Y$ be the restriction of the lift $K$ to $\{1\} \otimes \Omega[T]$. We claim that $z: \Omega[T] \rightarrow Y$ provides a lift in diagram (7).

Indeed, $\partial z=y$, and the deformation retraction $H$ gives a homotopy from $\operatorname{ir}(z)$ to $z$. This means that $i(x)$ and $\operatorname{ir}(z)$ are fiberwise homotopic to $z$, both via a homotopy which is given by $H$ when restricted to the boundary $\partial \Omega[T]$. But then there is a fiberwise homotopy between $\operatorname{ir}(z)$ and $i x$ which is constant on the boundary (using an argument similar to the proof of Lemma 2.5). It follows that $r(z)$ is homotopic (relative boundary) to $x$. Because $q: M \rightarrow X$ was a skeletal fibration, we conclude that $x=\phi^{*} r(z)$ for some automorphism $\phi$ of $T$.

Applying $q$, we see that $q(x)=\phi^{*} q r(z)=\phi^{*} q(x)$. But $X$ is a normal dendroidal set, so $\phi$ must be the identity automorphism. We conclude that $x=r(z)$, which means that $z: \Omega[T] \rightarrow Y$ provides a diagonal lift in diagram (7).

Corollary 3.7 Let $p: Y \rightarrow X$ be a fibration with normal domain. Then $p$ is a minimal fibration if and only if $p$ is a skeletal fibration. 
Proof All skeletal fibrations are minimal fibrations, so assume that $p$ is a minimal fibration. Then there is a trivial cofibration $i: M \rightarrow Y$ such that $p i$ is a skeletal fibration. By minimality of $p$, the map $i$ is an isomorphism and one finds that $p$ is skeletal.

Remark 3.8 In particular, the notion of skeletal fibration from Definition 3.1 is independent of the chosen cylinder, as long as it preserves colimits and has the properties mentioned in Section 2.4.

Corollary 3.9 Let $f: X^{\prime} \rightarrow X$ be a map of dendroidal sets with the property that for any element $x: \Omega[T] \rightarrow X^{\prime}$, the map $\operatorname{Aut}(x) \rightarrow \operatorname{Aut}(f x)$ is bijective. If $Y \rightarrow X$ is a minimal fibration with normal domain, then the base change $f^{*} Y \rightarrow X^{\prime}$ is a minimal fibration as well.

Proof This follows immediately from the corresponding property for skeletal fibrations: indeed, let $p: Y \rightarrow X$ be a skeletal fibration (with normal domain) and consider the following pullback square:

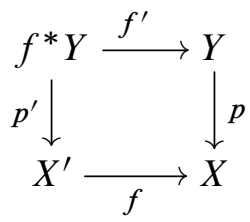

If $x, y: \Omega[T] \rightarrow f^{*} Y$ are fiberwise homotopic, then $f^{\prime} x$ and $f^{\prime} y$ are fiberwise homotopic as well. It follows that there is an element $\phi \in \operatorname{Aut}(T)$ such that $f^{\prime} x=\phi^{*} f^{\prime} y$. Projecting to $X$, we see $\phi$ is an automorphism of the element $p f^{\prime} x=f p^{\prime} x: \Omega[T] \rightarrow X$. By the assumption that $f$ induces a bijection on automorphism groups, it follows that $\phi^{*} p^{\prime} y=\phi^{*} p^{\prime} x=p^{\prime} x$. Since $Y^{\prime}$ is the pullback of $Y$ and $X^{\prime}$ over $X$, this implies that $x=\phi^{*} y$. We conclude that $Y^{\prime} \rightarrow X^{\prime}$ is indeed skeletal.

Example 3.10 The condition of Corollary 3.9 is satisfied by monomorphisms and by all maps between normal dendroidal sets (whose elements all have trivial automorphism groups). Furthermore, it is satisfied by all maps whose domain is a simplicial set, ie a dendroidal set without elements indexed by nonlinear trees. In particular, if $p: X \rightarrow S$ is a minimal fibration, then the fiber $X_{c}$ of $p$ over a color $c: \Delta[0] \rightarrow S$ is a minimal $\infty$-category.

Remark 3.11 Minimal (or skeletal) fibrations are not stable under base change along an arbitrary map. For example, consider the normal $\infty$-operad $X$ constructed in Example 3.2 and let $M$ be a skeletal deformation retract of it. The dendroidal set $M$ comes equipped with a 2-corolla $x: \Omega\left[C_{2}\right] \rightarrow M$ which is homotopic (relative to its boundary) to $\tau^{*} x$, where $\tau$ is the nontrivial automorphism of $C_{2}$. 
Now let $p: E_{\infty} \rightarrow *$ be a trivial fibration with normal domain. Then the map $M \rightarrow *$ is a skeletal fibration, but the base change $M \times E_{\infty} \rightarrow E_{\infty}$ is not. Indeed, let $y: \Omega\left[C_{2}\right] \rightarrow E_{\infty}$ be a lift of the unique map $\Omega\left[C_{2}\right] \rightarrow *$. Then the element $(x, y): \Omega\left[C_{2}\right] \rightarrow M \times E_{\infty}$ is fiberwise homotopic (rel boundary) to $\left(\tau^{*} x, y\right)$, but it is not related to $\left(\tau^{*} x, y\right)$ via an automorphism of $C_{2}$.

\section{Applications}

By way of example, we give two applications to the theory of left fibrations between dendroidal sets. Before stating these, we will recall the definitions of the relevant types of fibrations between dendroidal sets and the corresponding dendroidal horn inclusions.

\subsection{Fibrations between dendroidal sets}

Recall from Section 2 that every tree $T$ comes equipped with two kinds of face maps: inner face maps $\partial_{e}: T / e \rightarrow T$ contracting an inner edge $e$, and outer face maps $\partial_{v}: T / v \rightarrow T$ removing an outer vertex and all the outer edges attached to it. Each such face map determines a monomorphism of dendroidal sets $\partial_{\alpha}: \Omega[T / \alpha] \rightarrow \Omega[T]$ whose image we denote by $\partial_{\alpha} \Omega[T] \subseteq \Omega[T]$ and call the face opposite $\alpha$.

The union of all faces of $\Omega[T]$ is precisely the boundary $\partial \Omega[T]$ discussed in Section 2 . If $\alpha$ is an inner edge of $T$ or an outer vertex of $T$, we define the $\alpha$-horn of $\Omega[T]$, denoted by $\Lambda^{\alpha}[T]$, to be the union of all faces of $\Omega[T]$ except the face opposite $\alpha$. All horn inclusions $\Lambda^{\alpha}[T] \rightarrow \Omega[T]$ are normal monomorphisms, since $\Omega[T]$ is normal.

When $e$ is an inner edge, we will call $\Lambda^{e}[T] \rightarrow \Omega[T]$ an inner horn inclusion. A map of dendroidal sets is called an inner fibration if it has the right lifting property against all inner horn inclusions and is called inner anodyne if it has the left lifting property with respect to all inner fibrations. The pushout-product property for normal monomorphisms mentioned in Section 2.2 now admits the following refinement [8, Proposition 3.1]: if $A \rightarrow B$ is a normal monomorphism of dendroidal sets and $K \rightarrow L$ is a monomorphism of simplicial sets, then the pushout-product map

$$
L \otimes A \amalg_{K \otimes A} K \otimes B \longrightarrow L \otimes B
$$

is inner anodyne if one of the two maps is inner anodyne.

A dendroidal set $X$ is called an $\infty$-operad if the map $X \rightarrow *$ to the terminal object is an inner fibration. As we already remarked in Section 2.3 , the $\infty$-operads are precisely the fibrant objects of the operadic model structure on dendroidal sets. The fibrations in this model structure, ie the operadic fibrations, are all inner fibrations but not every 
inner fibration is an operadic fibration. For example, as we remarked in Section 2.3, the operadic fibrations between $\infty$-operads are those inner fibrations that also have the right lifting property against pushout-products of a normal monomorphism and an inclusion $\{i\} \rightarrow J$.

The left horn inclusions consist of the inner horn inclusions, together with the horn inclusions $\Lambda^{v}[T] \rightarrow \Omega[T]$ indexed by a leaf vertex of $T$ (in the case that $T$ is not a corolla) and the horns $\Lambda^{v}\left[C_{n}\right] \rightarrow \Omega\left[C_{n}\right]$ consisting of all faces indexed by a leaf of $C_{n}$. Because the other horns of the corolla play no role in our discussion, we will ignore the ambiguity in the notation $\Lambda^{v}\left[C_{n}\right]$.

A map of dendroidal sets is called a left fibration if it has the right lifting property against all left horn inclusions and left anodyne if it has the left lifting property with respect to all left fibrations. An inspection of the proof of [8, Proposition 3.1] shows that the pushout-product map (8) is left anodyne if either $A \rightarrow B$ is left anodyne or $K \rightarrow L$ is a left anodyne map of simplicial sets; see also Heuts [14, Appendix A.2]. It follows that left fibrations have the right lifting property against pushout-products of a normal monomorphism and the map $\{i\} \rightarrow J$, which is a left anodyne map of simplicial sets. In particular, all left fibrations over an $\infty$-operad are operadic fibrations.

As we already remarked in Section 2.3, for any dendroidal set $X$, the left fibrations $Y \rightarrow X$ form the fibrant objects of the covariant model structure on $\mathbf{d S e t} / X$. The fibrations in this model are, in particular, left fibrations, but the converse need not hold in general. An operadic weak equivalence of dendroidal sets $f: X \rightarrow X^{\prime}$ induces a Quillen equivalence $f_{!}: \mathbf{d S e t} / X \rightleftarrows \mathbf{d S e t} / X^{\prime}: f^{*}$ between the covariant model structures [14]. Applying this to an operadic weak equivalence $X \rightarrow X^{\prime}$ into an $\infty$-operad and using that left fibrations over $\infty$-operads are operadic fibrations, one can conclude that all left fibrations are operadic fibrations.

It follows that the covariant model structure on $\mathbf{d S e t} / X$ has the same cofibrations as the operadic model structure, but less fibrant objects. This means that the covariant model structure is a Bousfield localization of the operadic model structure; in particular, this implies that the covariant weak equivalences between left fibrations agree with the operadic weak equivalences.

\subsection{Gluing left fibrations}

Let $X$ be a simplicial set and let $A$ and $B$ be two subobjects of $X$ which cover $X$. The class of Kan fibrations satisfies a certain "homotopy descent" condition, which asserts that Kan fibrations over $A$ and $B$ can be glued, up to homotopy, to yield a fibration over their union $X$. More precisely, consider two Kan fibrations $Y_{A} \rightarrow A$ 
and $Y_{B} \rightarrow B$ and a homotopy equivalence between their restrictions to the intersection $A \cap B$. Then there exists a Kan fibration $Y \rightarrow X$ whose restrictions to $A$ and $B$ are homotopy equivalent to the original two fibrations.

This homotopy descent property is a special case of a result about homotopy colimits of simplicial sets stated in the work of Rezk [23], which is discussed by Puppe [21] in terms of topological spaces. It reflects the fact that Kan fibrations are local in nature: a map $Y \rightarrow X$ is a Kan fibration whenever its restriction to each simplex of $X$ is a Kan fibration. One may therefore expect a similar gluing result to hold for fibrations between dendroidal sets which have the same locality property. Operadic fibrations do not have this property, but left fibrations do since they are defined by the right lifting property with respect to left horns, which are subobjects of representables.

With this in mind, the homotopy descent property for left fibrations of dendroidal sets follows by a straightforward reduction to the situation where all left fibrations are minimal.

Proposition 4.1 Consider a diagram of dendroidal sets

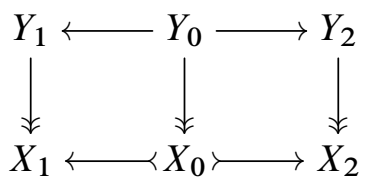

in which the vertical maps are left fibrations and the bottom horizontal maps are cofibrations. Suppose that both squares are "homotopy cartesian" in the sense that the maps $Y_{0} \rightarrow Y_{i} \times_{X_{i}} X_{0}$ are weak equivalences in the covariant model structure over $X_{0}$. Then there exists a left fibration over the pushout $X_{1} \cup_{X_{0}} X_{2}$, whose pullback to each of the $X_{i}$ is weakly equivalent to the left fibration $Y_{i} \rightarrow X_{i}$ in the covariant model structure over $X_{i}$.

Proof We can replace the above diagram by any weakly equivalent diagram of left fibrations over the $X_{i}$. In particular, we can assume that all dendroidal sets $Y_{i}$ are cofibrant.

We can further reduce to the case where all vertical maps are minimal left fibrations. Indeed, we can first replace diagram (9) by a diagram of the form

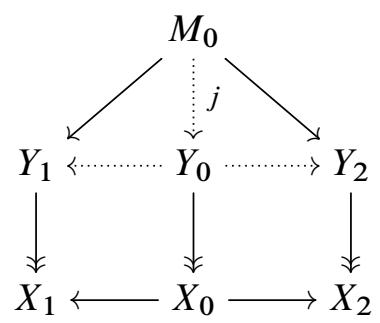


where $j$ is the inclusion of a minimal fibration with cofibrant domain. The resulting diagram of left fibrations remains homotopy cartesian. Next, replace this diagram by a diagram of the form

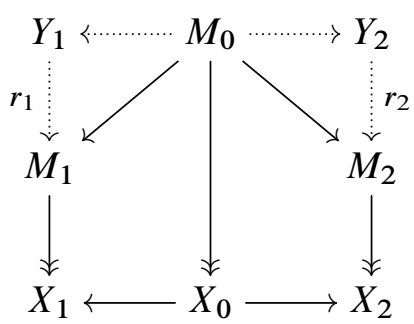

where $r_{1}$ and $r_{2}$ are fiberwise retractions onto minimal fibrations (with cofibrant domains). The vertical maps in the resulting diagram remain left fibrations and the maps $M_{0} \rightarrow M_{i} \times_{X_{i}} X_{0}$ are given by the composition

$$
M_{0} \longrightarrow Y_{0} \longrightarrow Y_{i} \times_{X_{i}} X_{0} \longrightarrow M_{i} \times_{X_{i}} X_{0} .
$$

The composition of the first two maps is an operadic weak equivalence, and the second map is the base change of a fiberwise deformation retract over $X_{i}$. It follows that the composite is a weak equivalence between two minimal fibrations over $X_{0}$, which means that it must be an isomorphism. In other words, the two solid squares in diagram (10) are both pullback squares.

Taking the pushout of the top and bottom row gives a map

$$
p: M_{1} \cup_{M_{0}} M_{2} \longrightarrow X_{1} \cup_{X_{0}} X_{2} .
$$

Because both squares in diagram (10) are cartesian, the pullback of this map to each of the $X_{i}$ reproduces the fibration $M_{i} \rightarrow X_{i}$, up to a canonical isomorphism. Since left fibrations between dendroidal sets are local, it follows that the map $p$ is a left fibration over $X_{1} \cup_{X_{0}} X_{2}$ whose pullback to each of the $X_{i}$ is weakly equivalent to the original left fibration $Y_{i} \rightarrow X_{i}$.

Proposition 4.1 has a simple model-categorical consequence, which we will now explain. The covariant model structures over all dendroidal sets assemble into a functor

$$
\text { Alg: } \text { dSet }^{\text {op }} \rightarrow \text { ModelCat }^{\mathrm{R}}, \quad X \mapsto(\operatorname{dSet} / X)^{\operatorname{cov}} .
$$

Given a diagram $X$ of dendroidal sets of the form

$$
X_{1} \stackrel{f}{\leftarrow} X_{0} \stackrel{g}{\rightarrow} X_{2},
$$


we thus obtain a diagram of (combinatorial, left proper) model categories and right Quillen functors between them:

$$
\operatorname{Alg}\left(X_{1}\right) \stackrel{f^{*}}{\longrightarrow} \operatorname{Alg}\left(X_{0}\right) \stackrel{g^{*}}{\longleftarrow} \operatorname{Alg}\left(X_{2}\right) .
$$

By Barwick [2], any such diagram of right Quillen functors admits a "homotopy pullback" model category $\operatorname{Alg}\left(X_{1}\right) \times_{\operatorname{Alg}\left(X_{0}\right)}^{h} \operatorname{Alg}\left(X_{2}\right)$, whose underlying category is the lax pullback of the above diagram of categories. More precisely, the homotopy limit model category has objects given by triples of objects $Y_{i} \in \operatorname{Alg}\left(X_{i}\right)$ together with two structure maps in $\operatorname{Alg}\left(X_{0}\right)$ :

$$
\alpha: Y_{0} \rightarrow f^{*} Y_{1} \text { and } \beta: Y_{0} \rightarrow g^{*} Y_{2} .
$$

The maps are maps of triples $Y_{i} \rightarrow Z_{i}$ that are compatible with the two structure maps. This category carries a model structure in which the trivial fibrations are triples of trivial fibrations $Y_{i} \rightarrow Z_{i}$, while the fibrant objects are given by triples of fibrant objects $Y_{i}$, together with structure maps $\alpha$ and $\beta$ which are weak equivalences.

In the present situation, where each of the categories $\operatorname{Alg}\left(X_{i}\right)$ is just the category of dendroidal sets over $X_{i}$, this means that the category underlying the homotopy pullback $\operatorname{Alg}\left(X_{1}\right) \times_{\operatorname{Alg}\left(X_{0}\right)}^{h} \operatorname{Alg}\left(X_{2}\right)$ is simply the overcategory

$$
\left(\text { dSet }^{1 \leftarrow 0 \rightarrow 2}\right) / X,
$$

whose objects are diagrams of shape (9). The model structure described above agrees with the model structure for which

- cofibrations are projective cofibrations between the underlying diagrams of dendroidal sets,

- fibrant objects are natural transformations $Y \rightarrow X$ such that each $Y_{i} \rightarrow X_{i}$ is a left fibration and each map $Y_{0} \rightarrow Y_{i} \times_{X_{i}} X_{0}$ is a covariant weak equivalence over $X_{0}$,

- weak equivalences between fibrant objects are degreewise weak equivalences.

This homotopy pullback model category comes equipped with a Quillen pair

$$
\text { colim: }\left(\text { dSet }^{1 \leftarrow 0 \rightarrow 2}\right) / X \rightleftarrows \text { dSet } / \text { colim } X \text { :pullback }
$$

to the covariant model structure over the pushout of the diagram $X$. The right adjoint sends a map over colim $X$ to its pullbacks to each of the $X_{i}$. Proposition 4.1 now has the following reformulation: 
Corollary 4.2 When $X_{1} \leftarrow X_{0} \rightarrow X_{2}$ is a diagram of cofibrations between dendroidal sets, the above Quillen pair is a Quillen equivalence.

Proof The derived unit is easily checked to be a natural weak equivalence, and the proof of Proposition 4.1 shows that the derived counit map is a natural weak equivalence.

Remark 4.3 The same result holds when only one of the two arrows is a cofibration. Indeed, this follows from the following facts: the operadic model structure is left proper [8], the covariant model structures over weakly equivalent dendroidal sets are Quillen equivalent, and two (naturally) Quillen equivalent diagrams of model categories have Quillen equivalent homotopy pullbacks [2].

\subsection{Weak equivalences between left fibrations}

As another application, we give an alternative, self-contained proof of the result of Heuts [14] that the weak equivalences between left fibrations of dendroidal sets are precisely the fiberwise weak equivalences.

Proposition 4.4 Let $p: X \rightarrow S$ be a left fibration between normal dendroidal sets. Then $p$ is a trivial fibration if and only if for every color $c: \eta \rightarrow S$, the fiber $X_{c}=X \times S \eta$ is a contractible Kan complex.

Corollary 4.5 Consider a map of left fibrations over $S$ :

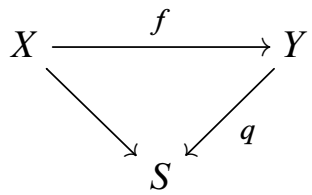

Then $f$ is a weak equivalence if and only if for every color $c: \eta \rightarrow S$, the map between fibers $X_{c} \rightarrow Y_{c}$ is a weak equivalence of Kan complexes.

Proof By Brown's lemma, weak equivalences between left fibrations are preserved by the right Quillen functor taking the base change along $c: \eta \rightarrow S$. A weak equivalence between left fibrations is therefore a fiberwise weak equivalence. For the converse, we can assume that $X$ and $Y$ are normal. Factor the fiberwise weak equivalence $f$ as a covariant trivial cofibration $X \rightarrow \tilde{X}$ followed by a covariant fibration $\tilde{X} \rightarrow Y$. Because $\tilde{X} \rightarrow S$ is a left fibration, the trivial cofibration $X \rightarrow \tilde{X}$ is a fiberwise weak equivalence. This implies that the covariant fibration $\tilde{X} \rightarrow Y$ is a fiberwise weak equivalence as well. 
For every color $c: \eta \rightarrow Y$, the fiber $\tilde{X} \times_{Y}\{c\}$ is isomorphic to the fiber over $\{c\}$ of the map between simplicial sets,

$$
\tilde{X} \times_{S}\{q c\} \longrightarrow Y \times_{S}\{q c\} .
$$

This map is a trivial fibration between Kan complexes because $\tilde{X} \rightarrow Y$ is a left fibration and a fiberwise weak equivalence. The left fibration $\tilde{X} \rightarrow Y$ has contractible fibers, so it follows from Proposition 4.4 that it is a trivial fibration.

The proof of Proposition 4.4 uses a more restrictive notion of homotopy, which is particularly useful in the setting of left fibrations. Recall that a linear order $[n]$ can be viewed as a tree with $n$ bivalent vertices (this induces the vertical inclusions in diagram (3)). We will denote the associated dendroidal set by $\Delta[n]$ and denote the tree associated to [0] by $\eta$. The following definition is taken from Cisinski and Moerdijk [8]:

Definition 4.6 For any tree $T$ and any number $k \geq 0$, let $\Omega[T] \star \Delta[k]$ be the dendroidal set represented by the tree $T \star[k]$ obtained by adding a vertex below the root of $T$ and grafting the result on top of the linear order $[k]$ :

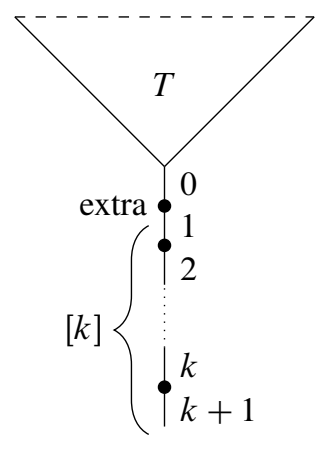

Each dendroidal set $\Omega[T] \star \Delta[k]$ comes equipped with an iterated degeneracy map $\sigma: \Omega[T] \star \Delta[k] \rightarrow \Omega[T]$ which removes the linear order and the extra vertex from $T \star[k]$. We will be particularly interested in the case where $n=0$, in which case the dendroidal set $\Omega[T \star \eta]=\Omega[T] \star \Delta[0]$ has three types of faces:

(0) the face $\partial_{0}: \Omega[T] \rightarrow \Omega[T \star \eta]$ associated to the inner edge marked by 0 in (11),

(1) the face $\partial_{1}: \Omega[T] \rightarrow \Omega[T \star \eta]$ opposite the root vertex, and

(2) for every face $\partial_{\alpha} \Omega[T] \rightarrow \Omega[T]$, a face $\partial_{\alpha} \Omega[T] \star \Delta[0] \rightarrow \Omega[T] \star \Delta[0]$.

The first two face maps for $k=0$ can be used to give another notion of homotopy between two elements of a dendroidal set, which is discussed in Moerdijk and Weiss [20]: 
Definition 4.7 Let $p: X \rightarrow S$ be a map of dendroidal sets and let $\beta, \beta^{\prime}: \Omega[T] \rightarrow X$ be two elements of $X$ with the same image in $S$. A fiberwise homotopy along the $0-e d g e$ from $\beta$ to $\beta^{\prime}$ is a commuting diagram

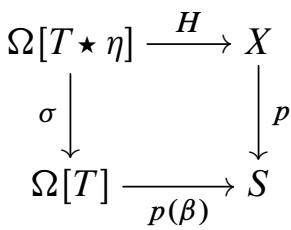

such that $\partial_{1} H=\beta$ and $\partial_{0} H=\beta^{\prime}$, and where $\sigma$ is the degeneracy removing the root vertex. Furthermore, we say that $H$ is a fiberwise homotopy relative to the boundary if the restriction of $H$ to each face of the form $\partial_{\alpha} \Omega[T] \star \Delta[0]$ factors over the degeneracy $\partial_{\alpha} \Omega[T] \star \Delta[0] \rightarrow \partial_{\alpha} \Omega[T]$.

Lemma 4.8 Let $p: X \rightarrow S$ be an operadic fibration and suppose that $H: \Omega[T \star \eta] \rightarrow X$ is a fiberwise homotopy along the 0 -edge between elements $\beta$ and $\beta^{\prime}$, relative to the boundary. Then $\beta$ and $\beta^{\prime}$ are fiberwise homotopic (relative to their boundary) with respect to the cylinder $J$ from Section 2.4.

Proof One can deduce the existence of such a homotopy either from Theorem B.2 in [8], or via the following argument. Observe that $H$ and the constant homotopy

$$
\Omega[T \star \eta] \stackrel{\sigma}{\longrightarrow} \Omega[T] \stackrel{\beta}{\longrightarrow} Y
$$

agree on all faces of $\Omega[T \star \eta]$, except for the face $\partial_{0}: \Omega[T] \rightarrow \Omega[T \star \eta]$ obtained by contracting the edge " 0 " above the root vertex (on which the value of $H$ was $\beta^{\prime}$, rather than $\beta$ ). Both of these homotopies therefore provide a diagonal lift for the same diagram

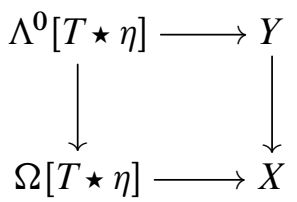

where the inner horn $\Lambda^{0}[T \star \eta]$ excludes the face that contracts the " 0 "-edge. But in the operadic model category, as in any model category with a choice of cylinder, lifts along inner horn inclusions are unique up to fiberwise $J$-homotopy, relative to the horn. In particular, we have that $\partial_{0} H=\beta^{\prime}$ and $\partial_{0}(\sigma \beta)=\beta$ are fiberwise $J$-homotopic relative to their boundary.

With this notion of homotopy at hand, we turn to the proof of Proposition 4.4. 
Proof of Proposition 4.4 Let $i: M \rightarrow X$ be the inclusion of a minimal fibration into $X$. The fibers of $p i: M \rightarrow S$ are weakly equivalent to those of $p: X \rightarrow S$ and the composite $p i$ is a trivial fibration if and only if the original fibration $p$ is a trivial fibration. We can therefore reduce to the case where $p: X \rightarrow S$ is a minimal fibration with normal codomain. Note that a minimal fibration with contractible fibers actually has trivial fibers; ie its fibers are isomorphic to $\eta$.

We will prove that any minimal left fibration $p: X \rightarrow S$ with trivial fibers is an isomorphism. It is immediate that $p$ induces a bijection on colors, and a left fibration inducing a surjection on colors is always an epimorphism. We show by induction that $p$ induces a monomorphism on all $n$-skeleta.

Assume that $p: X \rightarrow S$ induces an isomorphism on $(n-1)$-skeleta; let $\alpha: \Omega[T] \rightarrow S$ be a (possibly degenerate) element of degree $n$. We have to show that any two lifts of the element $\alpha$ to $X$, which exist by surjectivity of $p$, agree. The proof of this uses another inductive argument: we will say that an element $\alpha: \Omega[T] \rightarrow S$ has a trunk of height $0 \leq k \leq n$ if there is a factorization:

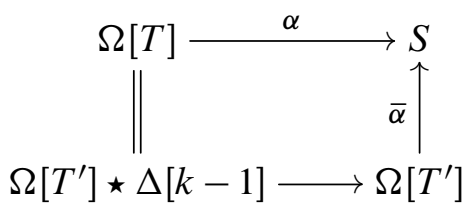

The left vertical isomorphism asserts that the tree $T$ has the form of picture (11), and the rest of the diagram asserts that $\alpha: \Omega[T] \rightarrow S$ factors over the degeneracy that collapses the linear order at the bottom of $T$. Note that having a trunk of height 0 is no condition, so it suffices to prove by decreasing induction on the number $k$ that $\alpha$ has a unique lift to $X$.

If $\alpha$ has a trunk of height $n$, then $\alpha: \Delta[n] \rightarrow S$ is a degenerate $n$-simplex in $S$. Such a simplex indeed has a unique (fully degenerate) lift since the fibers of $X \rightarrow S$ over each color are trivial. Now take $k<n$ and assume that all elements with a trunk of height $l>k$ have a unique lift.

Suppose that $\alpha: \Omega[T] \rightarrow X$ has a trunk of height $k$ and let $\beta, \beta^{\prime}: \Omega[T] \rightarrow X$ be two lifts of $\alpha$. Since $p$ induces an isomorphism on $(n-1)$-skeleta, the boundaries of $\beta$ and $\beta^{\prime}$ are the same. To conclude that $\beta$ and $\beta^{\prime}$ are the same, it suffices to show that $\beta$ and $\beta^{\prime}$ are fiberwise homotopic relative to their boundary, since we assumed that $p: X \rightarrow S$ was a minimal left fibration. By Lemma 4.8 it suffices to provide a fiberwise homotopy from $\beta$ to $\beta^{\prime}$ along the 0 -edge, relative to the boundary (see Definition 4.7). 
Pick any leaf vertex $v$ of the tree $T$. The idea will be to first construct a fiberwise homotopy $H$ which is constant on all faces except the face opposite $v$, and then to use our inductive hypothesis to conclude that this homotopy is also constant on this remaining face. We will construct the homotopy $H$ as a diagonal lift in a diagram

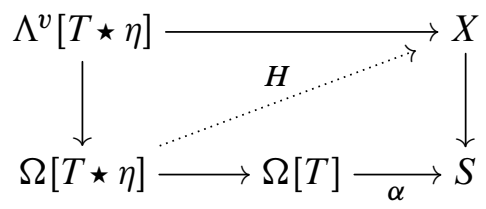

using the fact that $X \rightarrow S$ is a left fibration. In order to obtain this diagram, observe that the horn $\Lambda^{v}[T \star \eta]$ opposite the leaf vertex $v$ of $T \star \eta$ fits into a pushout square:

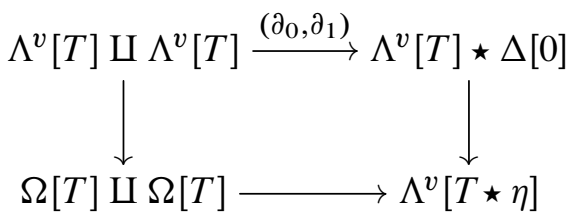

This follows immediately from the description of the faces of $\Omega[T \star \eta]$ given just above Definition 4.7. The elements $\beta$ and $\beta^{\prime}$, as well as the constant homotopy on the horn $\Lambda^{v}[T]$, define maps

$$
\Omega[T] \amalg \Omega[T] \stackrel{\left(\beta^{\prime}, \beta\right)}{\longrightarrow} X \quad \text { and } \quad \Lambda^{v}[T] \star \Delta[0] \stackrel{\sigma}{\longrightarrow} \Lambda^{v}[T] \stackrel{\Lambda^{v}(\beta)}{\longrightarrow} X
$$

that agree on $\Lambda^{v}[T] \amalg \Lambda^{v}[T]$. The induced map $\Lambda^{v}[T \star \eta] \rightarrow X$ out of the pushout fits into a commuting diagram of the form (12), whose diagonal $H: \Omega[T \star \eta] \rightarrow X$ provides a fiberwise homotopy between $\beta$ and $\beta^{\prime}$ which is constant on all faces except $\partial_{v} \Omega[T]$.

The restriction of $H$ to the remaining face $\partial_{v} \Omega[T] \star \Delta[0]$ gives a homotopy (rel boundary) from the face $\partial_{v} \beta$ to the face $\partial_{v} \beta^{\prime}$. This restriction fits into a commutative diagram:

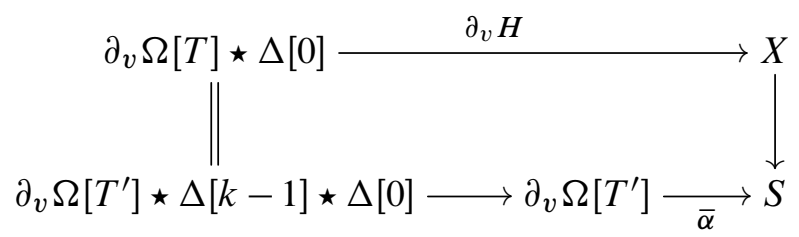

The left equality uses that the element $\alpha$ had a trunk of height $k$. In other words, the element $\partial_{v} H$ provides a lift of the degenerate element

$$
\partial_{v} \Omega\left[T^{\prime}\right] \star \Delta[k]=\partial_{v} \Omega\left[T^{\prime}\right] \star \Delta[k-1] \star \Delta[0] \longrightarrow \partial_{v} \Omega\left[T^{\prime}\right] \stackrel{\bar{\alpha}}{\longrightarrow} S,
$$


which is of degree $n$ and has a trunk of height $k+1$. But by the inductive assumption, elements with a trunk of height $k+1$ have unique lifts. It follows that $H$ is degenerate, which means that the homotopy $H$ is also constant on the remaining face $\partial_{v} \Omega[T]$.

The map $H: \Omega[T \star \eta] \rightarrow X$ thus provides a fiberwise homotopy (along the 0 -edge) between $\beta$ and $\beta^{\prime}$ which is constant on the boundary. Using Lemma 4.8 and the fact that $p: X \rightarrow S$ is a minimal fibration, this gives that $\beta=\phi^{*} \beta^{\prime}$ for some $\phi \in \operatorname{Aut}(T)$. Using $p$ to project to $S$, we find that $\phi$ induces an automorphism of the element $\alpha$. But $S$ was assumed to be normal, so $\phi$ is the identity, and $\alpha$ indeed has a unique lift.

\section{Other examples}

Recently Cisinski [7] has shown that the theory of minimal fibrations of simplicial sets can be generalized to model categories of presheaves over certain "Eilenberg-Zilber type" Reedy categories (see Section 2.1 of [7]), in which the cofibrations are the monomorphisms. Such Reedy categories share the combinatorial properties of the simplex category $\Delta$ that provide presheaves over them with a well-behaved skeletal filtration, the crucial tool used in the construction of minimal Kan fibrations.

The model structure on dendroidal sets does not entirely fit into this framework for the simple reason that the category $\Omega$ of trees is not a strict Reedy category. Our proof of Theorem 1.1 demonstrates how to take care of the automorphisms in $\Omega$ during the construction of minimal fibrations. To analyze the scope of our arguments, let us introduce the following notion of an "Eilenberg-Zilber type" generalized Reedy category, which is closely related to the notion of a skeletal category from Cisinski [6] and that of an EZ-category from Berger and Moerdijk [5]:

Definition 5.1 A generalized Reedy category $\mathbf{R}$ is called an Eilenberg-Zilber category if it satisfies the following two conditions:

(1) $\mathbf{R}^{-}$is the subcategory of split epimorphisms.

(2) Two maps $r \rightarrow s$ in $\mathbf{R}^{-}$are the same if they have the same set of sections.

Example 5.2 The category $\Omega$ of trees is an Eilenberg-Zilber category by Lemma 2.1. Apart from $\Omega$, the class of Eilenberg-Zilber categories includes well-known examples like the simplex category $\Delta$, Segal's category $\Gamma$ [25], Connes' cyclic category $\Lambda$ [11], the category of nonempty finite sets, and all group(oid)s.

Furthermore, the product of two Eilenberg-Zilber categories is also one, and for any presheaf $X$ on an Eilenberg-Zilber category $\mathbf{R}$, the category of elements $\mathbf{R} / X$ is again one. 
One can observe that all the definitions in Section 2 make sense when $\Omega$ is replaced by an arbitrary Eilenberg-Zilber category $\mathbf{R}$, as defined above. Although Lemma 2.2 need not hold for arbitrary $\mathbf{R}$, the corollary that elements are essentially unique degeneracies of nondegenerate elements still applies to normal presheaves over $\mathbf{R}$. It follows from this that any normal presheaf $X$ on an Eilenberg-Zilber category $\mathbf{R}$ admits a skeletal filtration, in which each inclusion $X^{(n)} \rightarrow X^{(n+1)}$ is a pushout of boundary inclusions $\partial \mathbf{R}[r] \rightarrow \mathbf{R}[r]$ into presheaves represented by objects $r \in \mathbf{R}$ of degree $n+1$; see Chapter 8 of Cisinski [6] for more details. Lemma 2.4 (which appears as Lemma 2.6 in [7] for strict Eilenberg-Zilber categories) shows that two degenerate elements of a normal presheaf $X$ are the same once their boundaries agree.

One can apply the arguments from Section 3 and proceed by induction along the skeletal filtration to prove the following generalization of Theorem 1.1(a):

Theorem 5.3 Let $\mathbf{R}$ be an Eilenberg-Zilber category and suppose that the category $\operatorname{PSh}(\mathbf{R})$ of set-valued presheaves on $\mathbf{R}$ carries a model structure in which the cofibrations are the normal monomorphisms. If $p: Y \rightarrow X$ is a fibration between cofibrant objects in this model structure, then $p$ admits a minimal fibration $M \rightarrow X$ as a fiberwise strong deformation retract.

Example 5.4 Apart from the model structures on dendroidal sets, there are many common model categories which are of this form. Examples include the model structure on the category $\operatorname{PSh}(\Lambda)$ of cyclic sets from Dwyer, Hopkins and Kan [12] and the model structure on the category PSh(Fin) of symmetric simplicial sets from Rosický and Tholen [24] (see also the erratum). One can produce many more examples by taking the category of simplicial presheaves over an Eilenberg-Zilber category $\mathbf{R}$ and equipping it with the generalized Reedy model structure [5] or any Bousfield localization thereof (viewed as a model structure for the Eilenberg-Zilber category $\mathbf{R} \times \Delta$ ). This includes the model structures on $\Gamma$-spaces and (dendroidal) Segal spaces as important examples.

Remark 5.5 Part (b) of Theorem 1.1 does not hold in general: it crucially relies on the fact that the category $\Omega / X$ is a strict Reedy category when $X$ is normal, in which case it is just a special case of Proposition 2.8 of [7]. This property is not shared, for example, by the category $\Gamma$ (or $\Gamma \times \Delta$ ).

In Theorem 5.3, the notion of a fiberwise strong deformation retract can be interpreted with respect to any model-categorical cylinder. However, the proof requires a cylinder construction that shares the good properties of the cylinder described in Section 2.4. More precisely, our proof in Section 3 needs a functorial factorization $X \amalg X:=$ $\{0,1\} \otimes X \rightarrow J \otimes X \rightarrow X$ of the fold map in $\operatorname{PSh}(\mathbf{R})$ such that: 
(i) The functor $J \otimes(-): \operatorname{PSh}(\mathbf{R}) \rightarrow \operatorname{PSh}(\mathbf{R})$ preserves all colimits.

(ii) For each $r \in \mathbf{R}$, the pushout-product map

$$
\{0,1\} \otimes \mathbf{R}[r] \cup_{\{0,1\} \otimes \partial \mathbf{R}[r]} J \otimes \partial \mathbf{R}[r] \longrightarrow J \otimes \mathbf{R}[r]
$$

is a normal monomorphism.

(iii) For each $r \in \mathbf{R}$ and $i=0,1$, the pushout-product map

$$
\{i\} \otimes \mathbf{R}[r] \cup_{\{i\} \otimes \partial \mathbf{R}[r]} J \otimes \partial \mathbf{R}[r] \longrightarrow J \otimes \mathbf{R}[r]
$$

is a trivial cofibration, where $\{i\} \otimes \mathbf{R}[r] \rightarrow J \otimes \mathbf{R}[r]$ is the inclusion of the relevant summand.

By [6, Proposition 8.1.35], the normal monomorphisms are the weakly saturated class on the boundary inclusions $\partial \mathbf{R}[r] \rightarrow \mathbf{R}[r]$, so the above three properties guarantee that the map $X \amalg X \rightarrow J \otimes X \rightarrow X$ provides a cylinder for all normal $X$.

Lemma 5.6 Let $\mathbf{R}$ be an Eilenberg-Zilber category and suppose that the category $\operatorname{PSh}(\mathbf{R})$ carries a model structure whose cofibrations are the normal monomorphisms. Then there exists a functorial factorization $X \amalg X \rightarrow J \otimes X \rightarrow X$ of the fold map in $\operatorname{PSh}(\mathbf{R})$ satisfying the above three properties.

Proof Since the functor $J \otimes(-)$ is required to preserve colimits, it suffices to produce the functorial factorization for representables. The fold map provides a natural transformation $\mathbf{R}[-] \amalg \mathbf{R}[-] \rightarrow \mathbf{R}[-]$ of $\mathbf{R}$-indexed diagrams in $\operatorname{PSh}(\mathbf{R})$. The category $\mathbf{R}$ is a generalized Reedy category, and $\operatorname{PSh}(\mathbf{R})$ carries a model structure with a set of generating cofibrations. The category of $\mathbf{R}$-indexed diagrams in $\operatorname{PSh}(\mathbf{R})$ therefore carries that part of the Reedy model structure (with respect to the given model structure on $\operatorname{PSh}(\mathbf{R})$ ) that allows us to factor this natural transformation as a Reedy cofibration $\mathbf{R}[-] \amalg \mathbf{R}[-] \rightarrow J \otimes \mathbf{R}[-]$, followed by a Reedy trivial fibration $J \otimes \mathbf{R}[-] \rightarrow \mathbf{R}[-]$. Property (ii) immediately follows from the fact that $\mathbf{R}[-] \amalg \mathbf{R}[-] \rightarrow J \otimes \mathbf{R}[-]$ is a Reedy cofibration.

Property (ii) immediately implies that the pushout-product map from property (iii) is a cofibration. To see that it is a weak equivalence, we proceed by induction on the degree of $r$. When $r=0$ the given map is a section of the map $J \otimes \mathbf{R}[r] \rightarrow \mathbf{R}[r]$, which was a trivial fibration by construction. Now suppose that property (iii) holds for all $r$ of degree $<n$, and let $s$ be of degree $n$.

This implies that the map $\{i\} \otimes X \rightarrow J \otimes X$ is a trivial cofibration for all those normal presheaves $X$ that can be obtained as iterated pushouts of boundary inclusions in degree 
less than $n$. This applies in particular to the boundary $\partial \mathbf{R}[s]$, so the first map in

$$
\{i\} \otimes \mathbf{R}[s] \longrightarrow\{i\} \otimes \mathbf{R}[s] \cup_{\{i\} \otimes \partial \mathbf{R}[s]} J \otimes \partial \mathbf{R}[s] \longrightarrow J \otimes \mathbf{R}[s]
$$

is a trivial cofibration. Since the composite is the section of the trivial fibration $J \otimes \mathbf{R}[s] \rightarrow \mathbf{R}[s]$, property (ii) also follows for elements $s$ of degree $n$.

Remark 5.7 Model categories of presheaves often have a cylinder provided by the socalled Lawvere interval [6, Example 1.3.9], ie by taking the product with the subobject classifier. This does not work in our setting since it gives a factorization of the fold map into a monomorphism, rather than a normal monomorphism, followed by a weak equivalence.

Finally, we would like to illustrate the use of the theory of minimal fibrations by means of the following application:

Example 5.8 Let us consider the following model for the homotopy theory of connective spectra parametrized by a simplicial set $S$. The category $(\mathbf{s S e t} / S)^{\Gamma^{\mathrm{op}}}$ of $\Gamma$-objects in sSet $/ S$ carries a model structure in which:

- The cofibrations are the normal monomorphisms.

- An object $X: \Gamma^{\mathrm{op}} \rightarrow \mathbf{s S e t} / S$ is a fibrant object if it is Reedy fibrant (with respect to the Kan-Quillen model structure on sSet $/ S$ ), and the Segal maps

$$
X(n) \longrightarrow X(1) \times{ }_{S} X(1) \times{ }_{S} \cdots \times{ }_{S} X(1)
$$

are trivial fibrations for all $n \geq 0$, as is the shear map $X(2) \rightarrow X(1) \times{ }_{S} X(1)$.

One can easily adapt the classical proof in simplicial sets (see eg [13]) to prove that any minimal object in this model structure is a locally trivial bundle of $\Gamma$-spaces, ie a $\Gamma$-space over $S$ whose pullback to a simplex $\Delta[n]$ is of the form $\Delta[n] \times F$, for some $\Gamma$-space $F$.

Each map of simplicial sets $S \rightarrow S^{\prime}$ induces a Quillen pair

$$
(\mathrm{sSet} / S)^{\Gamma^{\mathrm{op}}} \rightleftarrows\left(\mathrm{sSet} / S^{\prime}\right)^{\Gamma^{\mathrm{op}}}
$$

where the right adjoint pulls back a $\Gamma$-space over $S^{\prime}$ to a $\Gamma$-space over $S$. This Quillen pair is a Quillen equivalence whenever the map $S \rightarrow S^{\prime}$ is a weak equivalence. Associating to each simplicial set $S$ the above model category therefore provides a functor

$$
\text { sSet }^{\text {op }} \rightarrow \text { ModelCat }^{\mathrm{R}}, \quad S \mapsto(\text { sSet } / S)^{\Gamma^{\text {op }}} .
$$


A $\Gamma$-space over $S$ is fibrant precisely when its pullback to each simplex of $S$ is fibrant, since the Reedy fibrancy conditions and the Segal conditions require certain maps of simplicial sets over $S$ to be (trivial) Kan fibrations. In other words, being fibrant is a property "local" in $S$, in the sense of Section 4.2. We can therefore apply the proof of Proposition 4.1 to obtain the following variant of Proposition 1.2 for parametrized $\Gamma$-spaces:

Corollary 5.9 The functor (13) sending a simplicial set $S$ to the model category of $\Gamma$-spaces parametrized by $S$, sends a homotopy pushout of simplicial sets to a homotopy pullback of model categories.

This corollary remains true if one uses the covariant model structure on sSet $/ S$ rather than the Kan-Quillen model structure, since being fibrant requires certain maps of simplicial sets over $S$ to be (trivial) left fibrations, which is again a property local in $S$.

\section{References}

[1] M G Barratt, V K A M Gugenheim, J C Moore, On semisimplicial fibre-bundles, Amer. J. Math. 81 (1959) 639-657 MR

[2] C Barwick, On left and right model categories and left and right Bousfield localizations, Homology, Homotopy Appl. 12 (2010) 245-320 MR

[3] M Bašić, T Nikolaus, Dendroidal sets as models for connective spectra, J. K-Theory 14 (2014) 387-421 MR

[4] B van den Berg, I Moerdijk, W-types in homotopy type theory, Math. Structures Comput. Sci. 25 (2015) 1100-1115 MR

[5] C Berger, I Moerdijk, On an extension of the notion of Reedy category, Math. Z. 269 (2011) 977-1004 MR

[6] D-C Cisinski, Les préfaisceaux comme modèles des types d'homotopie, Astérisque 308, Société Mathématique de France, Paris (2006) MR

[7] D-C Cisinski, Univalent universes for elegant models of homotopy types, preprint (2014) arXiv

[8] D-C Cisinski, I Moerdijk, Dendroidal sets as models for homotopy operads, J. Topol. 4 (2011) 257-299 MR

[9] D-C Cisinski, I Moerdijk, Dendroidal sets and simplicial operads, J. Topol. 6 (2013) 705-756 MR

[10] D-C Cisinski, I Moerdijk, Note on the tensor product of dendroidal sets, preprint (2014) arXiv 
[11] A Connes, Cohomologie cyclique et foncteurs $\mathrm{Ext}^{n}, \mathrm{C}$. R. Acad. Sci. Paris Sér. I Math. 296 (1983) 953-958 MR

[12] W G Dwyer, M J Hopkins, D M Kan, The homotopy theory of cyclic sets, Trans. Amer. Math. Soc. 291 (1985) 281-289 MR

[13] P Gabriel, M Zisman, Calculus of fractions and homotopy theory, Ergeb. Math. Grenzgeb. 35, Springer, New York (1967) MR

[14] G Heuts, Algebras over infinity-operads, preprint (2011) arXiv

[15] A Joyal, The theory of quasi-categories and its applications, preprint (2008)

[16] J Lurie, Higher topos theory, Annals of Mathematics Studies 170, Princeton University Press (2009) MR

[17] J P May, Simplicial objects in algebraic topology, Van Nostrand Mathematical Studies 11, D. Van Nostrand Co., Princeton, NJ (1967) MR

[18] I Moerdijk, Lectures on dendroidal sets, from "Simplicial methods for operads and algebraic geometry” (C Casacuberta, J Kock, editors), Springer, New York (2010) 1-118 MR

[19] I Moerdijk, I Weiss, Dendroidal sets, Algebr. Geom. Topol. 7 (2007) 1441-1470 MR

[20] I Moerdijk, I Weiss, On inner Kan complexes in the category of dendroidal sets, Adv. Math. 221 (2009) 343-389 MR

[21] V Puppe, A remark on “homotopy fibrations”, Manuscripta Math. 12 (1974) 113-120 MR

[22] D G Quillen, The geometric realization of a Kan fibration is a Serre fibration, Proc. Amer. Math. Soc. 19 (1968) 1499-1500 MR

[23] C Rezk, Fibrations and homotopy colimits of simplicial sheaves, preprint (1998) arXiv

[24] J Rosický, W Tholen, Left-determined model categories and universal homotopy theories, Trans. Amer. Math. Soc. 355 (2003) 3611-3623 MR Correction in 360 (2008) 6179

[25] G Segal, Categories and cohomology theories, Topology 13 (1974) 293-312 MR

Mathematical Institute, Utrecht University, PO Box 80010, 3508 TA Utrecht, Netherlands

i.moerdijk@uu.nl, j.j.nuiten@uu.nl

Received: 3 December 2015 Revised: 5 April 2016 